\title{
CRISPR/Cas9-Based Genome Editing in filamentous fungus Glarea lozoyensis and its application in manipulating gloF
}

Teng-Yun Wei, Yuan-Jie Wu, Qiu-Ping Xie, Jia-Wei Tang, Zhi-Tuo Yu, Song-Bai

Yang, Shao-Xin Chen*

Shanghai Institute of Pharmaceutical Industry, China State Institute of Pharmaceutical Industry, 285 Gebaini Road, Pudong, Shanghai 201203, China

Email: Prof. Dr. S.-X. Chen sxzlb@263.net

Table S1. List of guide sequences and protospacer adjacent motif (PAM) sequences of each target locus in this study

\begin{tabular}{|c|c|c|}
\hline Target locus & Guide sequence & PAM \\
\hline gloA & ccttctcgtcatccagcetg & CGG \\
\hline$g l o C$ & tcgtagaaagattccagcca & TGG \\
\hline$g l o F$ & catcgtggttggagtgctgt & CGG \\
\hline
\end{tabular}

Table S2. Strains and plasmids used in this study

\begin{tabular}{|c|c|c|}
\hline Strains or plasmids & Description & Source \\
\hline $\begin{array}{l}\text { Glarea lozoyensis } \\
\text { SIPI1208 }\end{array}$ & $\begin{array}{l}\text { An industrial producing strain for pneumocandin } B_{0} \text { mutated } \\
\text { from ATCC } 74030\end{array}$ & SIPI* \\
\hline E. coli $\mathrm{DH} 5 \alpha$ & $\begin{array}{l}\text { F-, } \varphi 80 \text { dlacZ } \Delta M 15, \Delta(\text { lacZYA-argF) U169, deoR, recA1, } \\
\text { endA1, hsdR17 (rk-, mk+), phoA, supE44, } \lambda \text {-, thi-1, gyrA96, } \\
\text { relA1 }\end{array}$ & CWBIO \\
\hline A. tumefaciens LBA4404 & Ach5 (rifR) Ti pAL4404 (strepR) Octopine & WEIDI \\
\hline G. lozoyensis (PC) & G. lozoyensis integrated with cas 9 expression cassette & This study \\
\hline G. lozoyensis (sgRNA) & $\begin{array}{l}\text { G. lozoyensis integrated with sgRNA expression cassette, } \\
\text { sgRNA was used to target } g l o A\end{array}$ & This study \\
\hline Gl-ap-htyE & G. lozoyensis integrated with ap-htyE expression cassette & This study \\
\hline$G l(P C)-a p-h t y E$ & gloF gene was replaced with ap-htyE & This study \\
\hline $\mathrm{pDHt} / \mathrm{sk}-\mathrm{PC}$ & plasmid containing cas 9 expression cassette & 1 \\
\hline pAg1-H3 & $\begin{array}{l}\text { vector for AMT transformation of fungus containing hyg } \\
\text { gene as selection marker }\end{array}$ & 2 \\
\hline $\mathrm{pAgG}$ & vector for AMT transformation of fungus containing neo & This study \\
\hline
\end{tabular}




\begin{tabular}{|l|l|l|}
\hline & gene as selection marker & \\
\hline pAgG-sgRNA-gloA & pAgG with specific sgRNA targeting gloA & This study \\
\hline pAgG-sgRNA-gloC & pAgG with specific sgRNA targeting gloC & This study \\
\hline pAgG-sgRNA-gloF & pAgG with specific sgRNA targeting gloF & This study \\
\hline pAgG-Pglgpd-Tglgpd & pAgG with glgpd promoter and terminator used for the & This study \\
\hline pAgG-ap-htyE & expression of exogenous genes in G. lozoyensis & \\
\hline pAgG-sgRNA-gloF-ap- & plasmid for the expression of ap-htyE in G. lozoyensis & This study \\
\hline$h t y E$ & the 5' and 3' flanking regions & This study \\
\hline
\end{tabular}

*Microbiological Culture Collection Center of Shanghai Institute of Pharmaceutical industry

Table S3. Primers used in this study

\begin{tabular}{ll}
\hline Primers & Sequences $\left(5^{\prime}-3^{\prime}\right)$ \\
\hline PtrpC-F/R & acccAAGCTTgggaatcgatgatcaggcctcgac \\
& aatccatcttgttcaatcatttggatgcttgggtagaata \\
& tattctacccaagcatccaaatgattgaacaagatggatt \\
NeoR-F/R & gatcccggtcggcatctacttcagaagaactcgtcaagaa \\
& ttcttgacgagttcttctgaagtagatgccgaccgggatc \\
TtrpC-F/R & ctggactagtcettcgtccggcgtagaggatcct \\
& gttgtaaaacgacggccagtgtgtcacttcgcgtcttgtc \\
Pgpd-F/R & ccgtcgacagcgctactagtattgttttctggtgaagattagcc \\
& actagtagcgctgtcgacggtcttcaccactcatttctca \\
Tgpd-F/R & tcctctacgccggacgaaggagtgagtcgatggcgaaatcg \\
& aatcttcaccagaaaacaatatggctatcactacgctaga \\
ap-htyE-F/R & agaaatgagtggtgaagaccgttacccataggtcctcgtca \\
sgRNA-R-2 & gACTAGtcgggggatcctctagatcttctgcaggtcgactctagag \\
sgloA-F & GTATGAAAGGACGAAACACCcttctcgtcatccagcctggttttagagctagaaatagc \\
sgloC-F & GTATGAAAGGACGAAACACCgcctcctgtattcagtaggcgttttagagctagaaatagc \\
sgloF-F & GTATGAAAGGACGAAACACCcatcgtggttggagtgctgtgttttagagctagaaatagc \\
& gttgtaaaacgacggccagtgcttcatttgatcgatgttcc \\
htyE-F/R & GGTGTTTCGTCCTTTcatacgacagtaggtattcgc \\
\hline & atggctatcactacgctaga \\
\hline
\end{tabular}




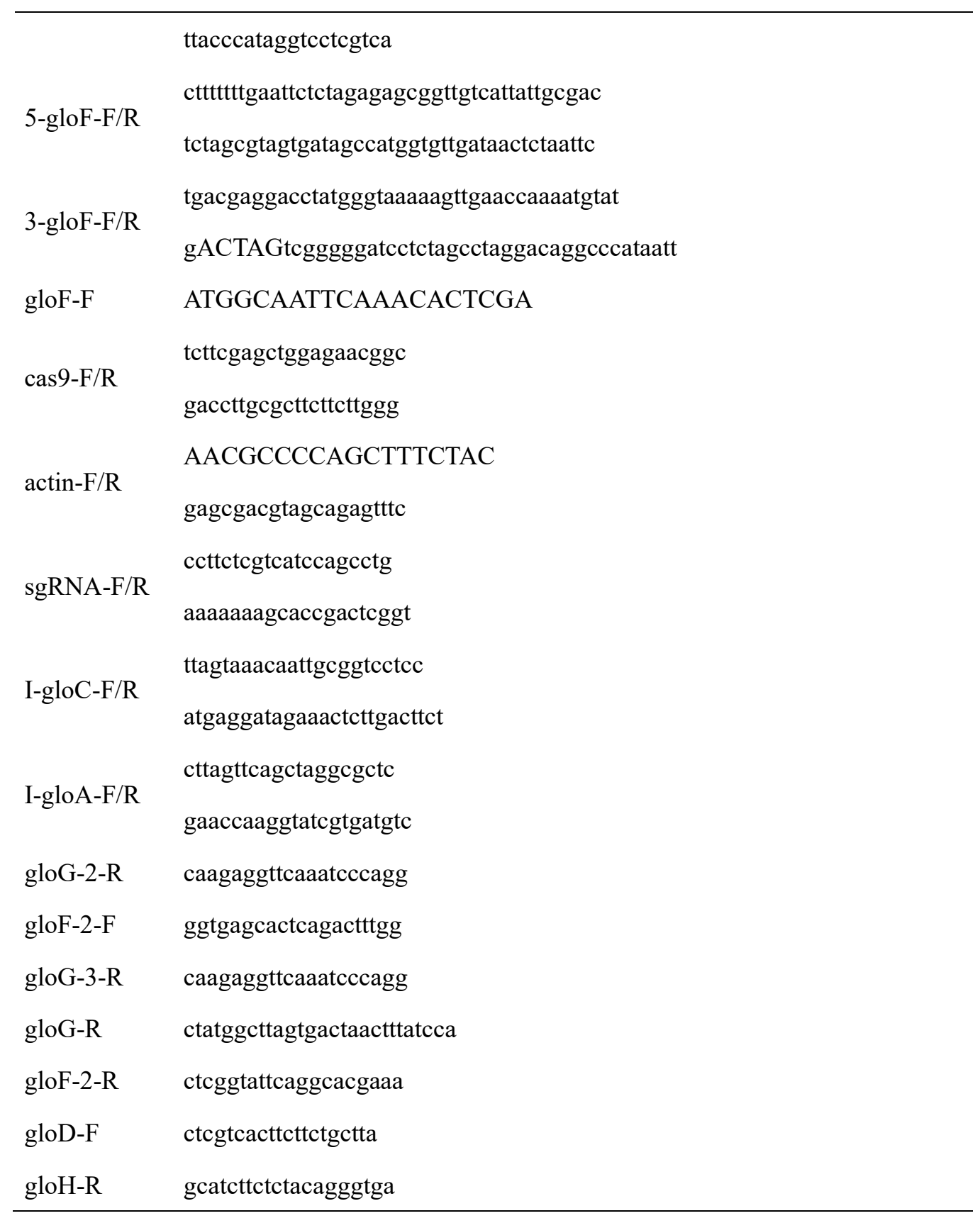




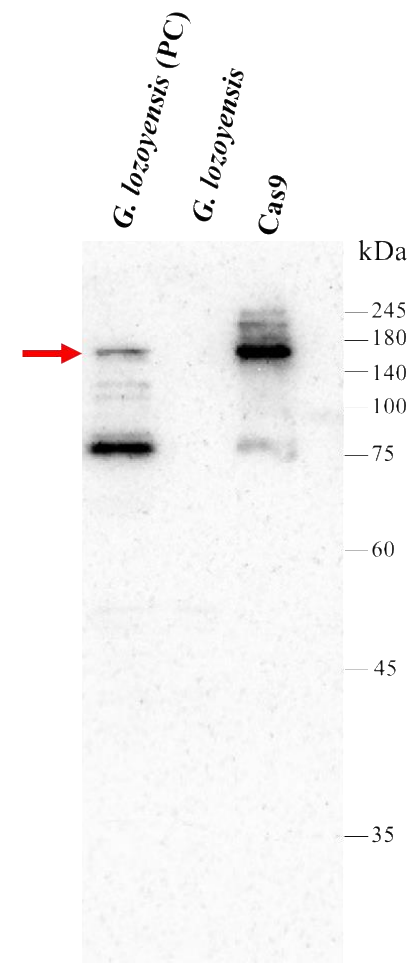

Figure S1. Detection of Cas9 expression in G. lozoyensis (PC) by western blot. Lanes G. lozoyensis (PC) and G. lozoyensis were proteins extracted from strain G. lozoyensis (PC) and G. lozoyensis respectively. Lane Cas9 was purified protein (Genscript). The proteins extracted from G. lozoyensis were used as negative control. The purified Cas9 was used as positive control. $50 \mu \mathrm{g}$ of total protein of the indicated strains was probed with Anti-Cas9 antibody (Abcam). The red arrow indicated the site of Cas9. 
a

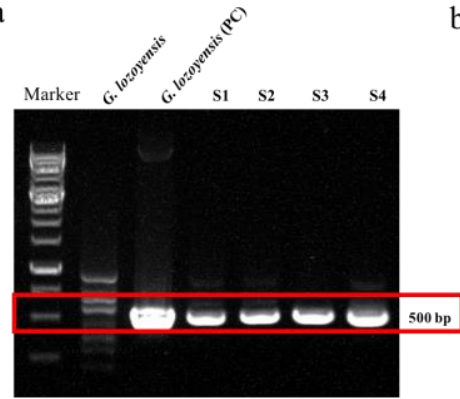

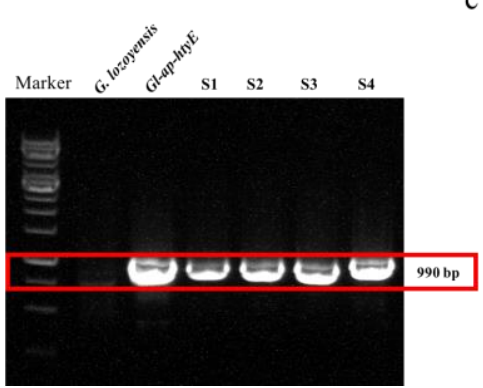

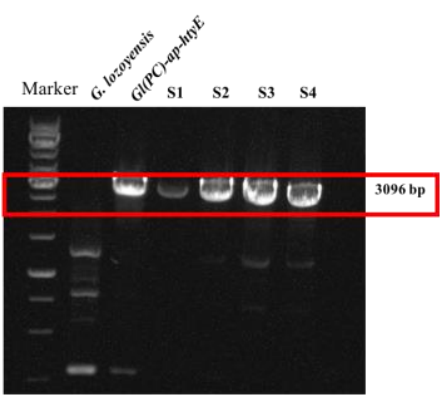

Figure S2. (a) Investigation of the stability of cas9 gene in G. lozoyensis (PC) by PCR. G. lozoyensis (PC) was cultured 4 times consecutively. The primer piars are cas9-F/R. Line G. lozoyensis is negative control amplified from G. lozoyensis gDNA; Line G. lozoyensis (PC) is positive control amplified from G. lozoyensis (PC) gDNA; Line S1-S4 are amplified from consecutive subcultures. (b) Investigation of the stability of $a p-h t y E$ gene in $G l-a p-h t y E$ by PCR. Gl-ap-htyE was cultured 4 times consecutively. The primer piars are htyE-F/R. Line G. lozoyensis is negative control amplified from G. lozoyensis gDNA; Line Gl-ap-htyE is positive control amplified from Gl-ap-htyE gDNA; Line S1-S4 are amplified from consecutive subcultures. (c) Stability investigation of the insertion of $a p-h t y E$ gene in $G l(P C)$-ap-htyE by PCR. $G l(P C)$-ap-htyE was cultured 4 times consecutively. The primer piars are gloD-F/htyE-R. Line G. lozoyensis is negative control amplified from $G$. lozoyensis gDNA; Line Gl-ap-htyE is positive control amplified from $G l(P C)$-ap-htyE gDNA; Line S1-S4 are amplified from consecutive subcultures. 
a

\begin{tabular}{|c|c|}
\hline \multirow[t]{2}{*}{$\begin{array}{l}\text { GloF } \\
\text { Ap-HtyE }\end{array}$} & $\begin{array}{l}\text { MAIQTLDYRDFQYGGQEQHRTFCHNLCETLSTWGFIKIQNTSIPDAVIDELFSYNKKFFA } \\
\text { MAITTLDFNQFRSTSADERQIFCADLCETLSVYGFAKIRNTTLSNELIDEIFKYTRSFFA }\end{array}$ \\
\hline & $* * * * * *: .: *: \quad,::::: * *: * * * * * * .: * * * * * * *:: .::: * * *: * . * .:, * * * *$ \\
\hline \multirow[t]{2}{*}{$\begin{array}{l}\text { GloF } \\
\text { Ap-HtyE }\end{array}$} & $\begin{array}{l}\text { LPEHIKQKARHPAAPNPHRGWSAVGQEQLSRIAGFEKDEETDGFVPEYRESFDQGAADDE } \\
\text { LPNDIKAKAKHPNAPNPHRGWSAIGQERVWKISGFEQNKERTDSYNEFRESFDQGAADDQ }\end{array}$ \\
\hline & **: :** **:*** **********:***:: :*:***:::*..$\quad *: * * * * * * * * * * *:$ \\
\hline \multirow[t]{2}{*}{$\begin{array}{l}\text { GloF } \\
\text { Ap-HtyE }\end{array}$} & $\begin{array}{l}\text { LFPNRWIDEDDLPGFRKFMENYYEMCYNFHTQLLRAISTGLSLPEDLLLSRHQTDTSELR } \\
\text { LFPNRWVDEDDLPGFQAFMEGFYKSCDELHAHLLRAISTGLKLPDTLLPSKHRHNTSELR }\end{array}$ \\
\hline & $* * * * * *: * * * * * * * *: * * * .: *: \quad *::: *:: * * * * * * * * * . * *: \quad * * *: *::: * * * * *$ \\
\hline \multirow[t]{2}{*}{$\begin{array}{l}\text { GloF } \\
\text { Ap-HtyE }\end{array}$} & $\begin{array}{l}\text { MNHYPAIACENLKFGMRIGEHSDFGTLTLLLQDSTGGLQVEDQKKLGTFIPVESDSRYEV } \\
\text { LLHYPPIPCSALRSNMRIGEHSDFGTLTLLLQDSVGGLQVEDQRNPRSFIPVEPEDGYEV }\end{array}$ \\
\hline & 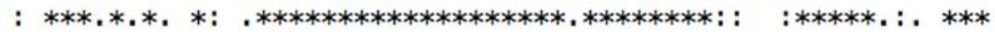 \\
\hline \multirow[t]{2}{*}{$\begin{array}{l}\text { GloF } \\
\text { Ap-HtyE }\end{array}$} & $\begin{array}{l}\text { IVNVGDCLQRWTNRRLRSANHRVHLPEGKNFKSDEVLADRYSVAYFGKPDRNVLVDSFPE } \\
\text { VINIGDCLQRWTNRRLCSANHRVMLPEGKDVDSEEVLDDRYSVAYFGKPDRDVLVDTLPE }\end{array}$ \\
\hline & 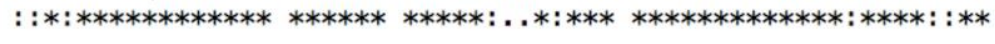 \\
\hline $\begin{array}{l}\text { GloF } \\
\text { Ap-HtyE }\end{array}$ & $\begin{array}{l}\text { FCRGGE-SKYNDHMNALEYNQTKLLRTYA } \\
\text { CVEVGERVEYGDHLTALQYNQIKLTRTYG }\end{array}$ \\
\hline & . ** $: *, * *:, * *: * * * * * * * *$, \\
\hline
\end{tabular}

b

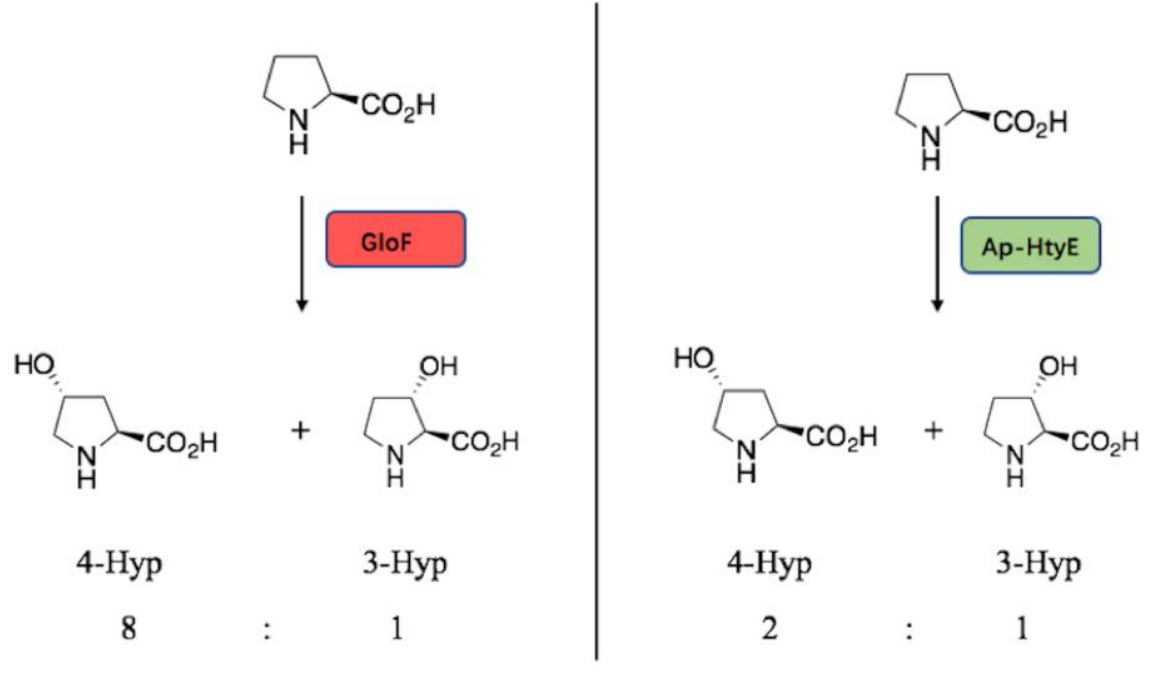

Figure S3. (a) amino acid sequence alignment of GloF (XP_008078282) and Ap-HtyE

(AFT91391.1); (b) 4-Hyp/3-Hyp ratios from conversions of Pro with Ap-HtyE and GloF. ${ }^{3}$ 
A. nidulans-GPD

G. lozoyensis-GPD

A. nidulans-GPD

G. lozoyensis-GPD

A. nidulans-GPD

G. lozoyensis-GPD

A. nidulans-GPD

G. lozoyensis-GPD

A. nidulans-GPD

G. lozoyensis-GPD

A. nidulans-GPD

G. lozoyensis-GPD
MAP-KVGINGFGRIGRIVFRNAIEAGTVDVVAVNDPFIETHYAAYMLKYDSQHGQFKGTI MAPTKVGINGFGRIGRIVFRNAIEHDDIDIVAVNDPFIEPHYAAYMLKYDSTHGQFKGDI **** ************************, : $;: * * * * * * * * *, * * * * * * * * * * * * * * * * * * *$

ETYDEGLIVNGKKIRFHTERDPANIPWGQDGAEYIVESTGVFTTQEKASAHLKGGAKKVV KATDNGLEVNGKHIRFYTERDPANIPWSETDAYYIVEATGVFTTTDKAKAHLKGGAKKVV

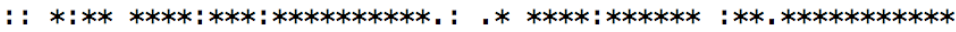

ISAPSADAPMFVMGVNNETYKKDIQVLSNASCTTNCLAPLAKVINDNFGI IEGLMTTVHS ISAPSADAPMYVMGVNEKTYKSDIEVISNASCTTNCLAPLAKVINDEFTIIEGLMTTIHS

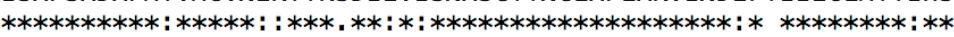

YTATQKVVDGPSAKDWRGGRTAATNIIPSSTGAAKAVGKVIPSLNGKLTGMAMRVPTSNV YTATQKTVDGPSAKDWRGGRTAAQNIIPSSTGAAKAVGKVIPELNGKLTGMSMRVPTSNV

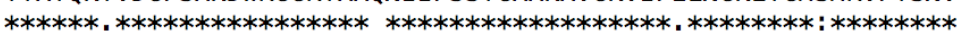

SVVDLTVRTEKAVTYDQIKDAVKKASENELKGILGYTEDDIVSTDLNGDTRSSIFDAKAG SVVDLTCRLEKGASYDTIKAAIKKAADGPLKGILAYTEDEVVSTDLNGDTHSSIFDAKAG ******* * **, : :** ** *:***: : , ******,****::**********:**********

IALNSNFIKLVSWYDNEWGYSRRVVDLITYISKVDAQ-

ISLNDNFVKLVAWYDNEWGYSRRVLDLLSYISKVDAGK

$*: * * ; * *: * * *: * * * * * * * * * * * *: * *:: * * * * * * *$

Figure S4. Glyceraldehyde-3-phosphate dehydrogenase amino acid sequence alignment of $G$.

lozoyensis (XP_008086288.1) and A. nidulans (P20445.2)

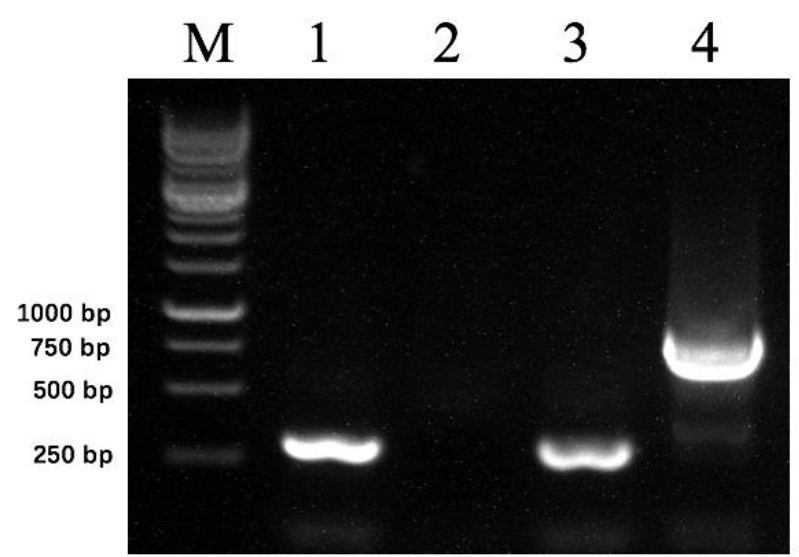

Figure S5. RT-PCR analysis of $g l o F$ and $a p-h t y E$ in Gl-ap-htyE. Line 1 and 3 are amplified by gloF-F/gloF-2-R primer pairs. Line 2 and 4 are amplified by htyE-F/R pairs. Line 1 and 2 are $G$. lozoyensis cDNA; Line 3 and 4 are Gl-ap-htyE cDNA. 


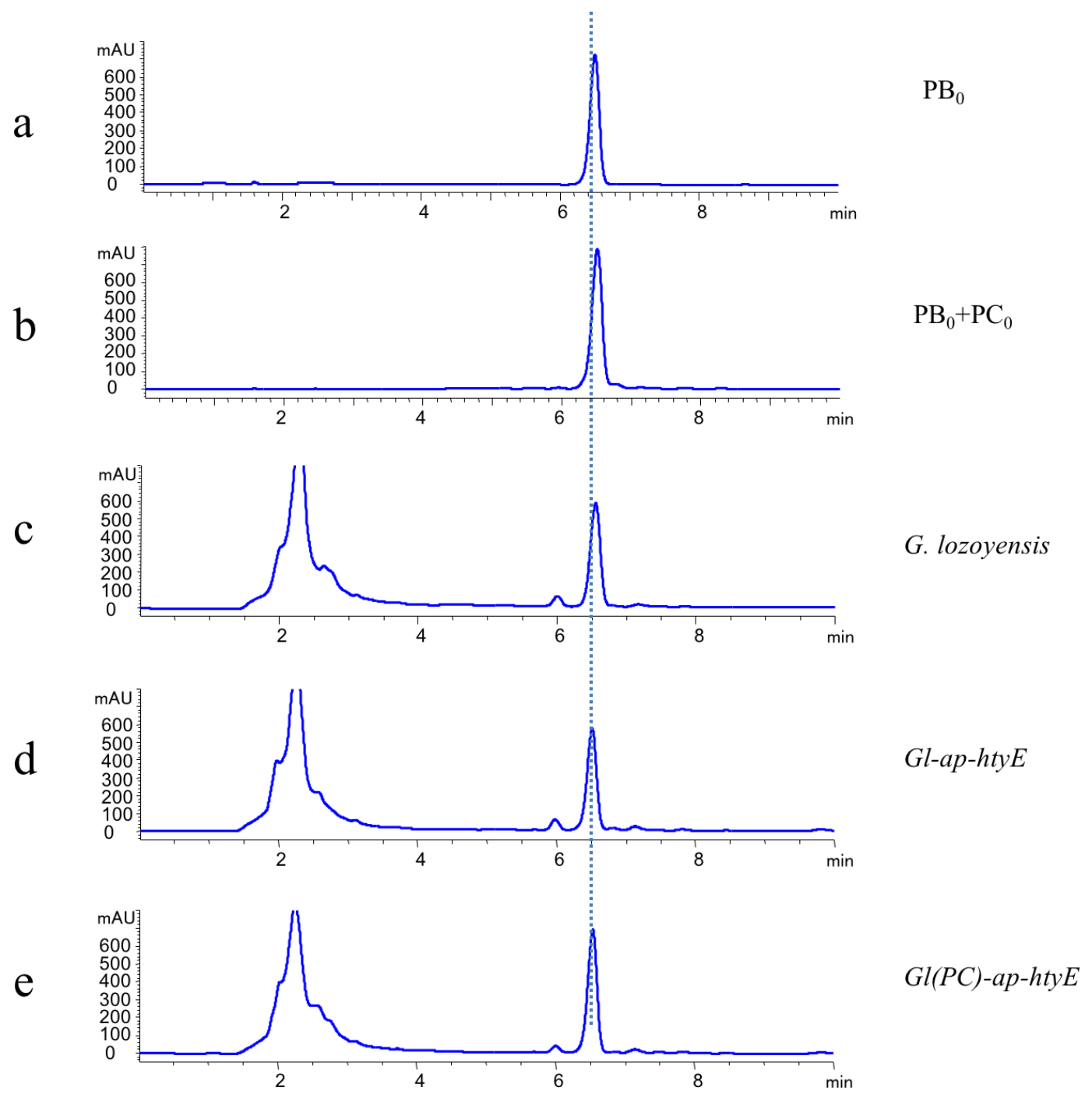

Figure S6. RP-HPLC profiles of pneumocandin $\mathrm{B}_{0}$ standard (a), a mixture of $\mathrm{PB}_{0}$ and $\mathrm{PC}_{0}(\mathrm{~b})$, fermentation extracts of the original strain G. lozoyensis (c), fermentation extracts of engineering

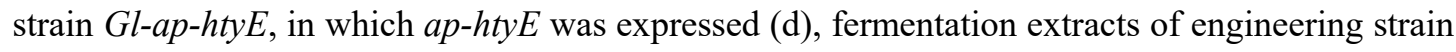
$G l(P C)-a p-h t y E$, in which $g l o F$ was replaced with ap-htyE (e). 


\section{Pglgpd sequence (2 kb)}

tgtcacttcgcgtctttgtctgttacacgatacagcaaactttaagaatgaacctttctggcggctcctaaatcaatgaaagacg gactcggacccaatgaggccaacaacggaagttggtacgtttcagggccccaaagtatccttcgctccatgatatgaatgtc tagatttagcgactctctaaccaagaatatctacggtctgacctagtgcagaacaatgtattcgtgaaaggaaagattgaaac attctgcggcagcaataaggcagctagttctactaacgttaaactgaaagcgaagggatttgaaagttgatgaacagttgtaa gtgacacagtaaatccttaagagcctaagatatgatattgtggtttgaaagaagttattttctatgtaagcacgtaaaacagcg aaagatcaaagactattgtggcattagatctttgttagaaagaagtacattttgtttggcgaagagatgtgagcataaaagggt gaatgaaagatgtatgaatggttgtccggcagaatcagatgtggatttctgctggtgaacccgtaagagaaagcctcacgtg tctccegatcataaatccataacagttccaaaaaattcatgagcgegagcgegagcggcaataaatcagctttttcaagatt gtcacgagttatgaagccatgcaggtttccgagttttcaacacattctgttgtataatcatcgatgcatagcacgtgatttctgg ctcgaaagcaaacaagattgggaggctttgggcagctctttatctggcgagaaatctgaatgagaatgcttctcgtgcgaaa gaataacgctgttggcaattagagggcgaattcagccacctgcaatgacaataggagcttagcttcaagtcagataaaagg cgtggggcgttatcgagaataagaaaagcccgaagatttggccggctgccgttaaatatttgtcaagcaaaaggcaggga atgagtgttactcatatggattgagggaataacactttgcaagaaggatgccatgcaatgagaaaagtctgaactacacgtg gcggcgcaacgcaacgetccgcagttaggaggaggtttagctgacagcgcagtctggagacaccgagaggaaatgttc ctgttgacgatggagcttccattttgaatcttcaaggggggttggtgttctggagtttgatattggtatggtcaggaccacgag aaacagattacctaggtaaagaataatagcttcgccgttcgaacgatagctcggttaaagaattacttccattctatccgaatta tcgtggagtatctagttcctcatagcaccgtatctccceggcggctcggagcgagtcgcetgctcttaaagtgtgacgtgatg gtgactctgctcactctgctcactctgctcactcctccagcctcatcgactgcaggtggtggtgagactgtattattgagagatt taaatctccgtcagcttttcctgatcatctggagcgaaaggaataaataaaaacttcgtaaagcgtaacgggaacacgattgc gcaggggcgcgaccggatgcacgataagatgagtgaggaagccaattgaggactcatccactccacgtcgagaaaaat catcatctagtctgtggtgtttccagctggttggttgccagacagccttgtcgcagtgattgcttgcttggtttcggtggtcgtg gtcgtggcttgcccetcttttcegtagcgtagcgcatcttcccatctcaacaacacccaccacgagcacaaactctctcatttt cgtctcgattcttcttccttcgacatcgtacgaaacaaccaacctaagaaaaacaatcaacgaaacatgtatgtatatccttccc catctgttcactctgccagtcgeccatcctctacatcgagaattcttgtcgctccttcgtcatcgeggggetaatcttcaccaga aaacaat

\section{Tglgpd sequence (481 bp)}

ggtcttcaccactcatttctcacatttgtatcaatggtatcgctttctgattctcttttccctggcagagaagtgctgaaaggtaa actttaaaggggaatgaacaaggatagacatctttacatacaatgattgacggcacaccaatgcctttacgatagcatgaaa atagaagtcctcaattgagtgtatcatcctctcagattttcatacatgactctttgcaatttgcatgttcctcgtgctagacaagag cagtttcagtgccaaaaagatctttcacccacttccttaaactgctttgttatgatgagaacacaggetgtccagtggcattccc tttcggtctcctccatgttaatatgcaaatgtacttcgtacgagatctaccettaccegetcctttgacaaacctacatgecetgtt 
tttgccatactgctcttctcctactatgttctgcgatttcgccatcgactcac

sgRNA promoter sequence (559 bp)

gcttcatttgatcgatgttccaacacaaatgacactcgectacgtattacaaccaactctctagcaactaactgccaaacactct atcgaacttagtcgagcagtccgtctgaagttgattcattaagagtaacagactgccttgaatctctcaactcagcatttacca agaaagcgttctaaaatccgtaccacacgecgtcatttctgacataacttgaatcggectcccatcacgtgacgcaccecga ctcccttaacaaccgcacaagtcctcacacaccacaacccctcaaccacacaaccaccettcttccaccaaaacacaattt ccctacaaactcatatagctgtgtttgtgtttctctcatcacaccccccegctcttttttcagccetccagcgtcaactatataaat ctaaaaccacccaccttgtcacatacgatcatagactgatgagaattgggcatccegtccgetctgecacacacaagcatc agatcggtagattagtagttgggtgggtgaccaccagcgaatacctactgtcgtatg

5s rRNA sequence was underlined.

\section{References}

(1) Liu, R., Chen, L., Jiang, Y., Zhou, Z., and Zou, G. (2015) Efficient genome editing in filamentous fungus Trichoderma reesei using the CRISPR/Cas9 system, Cell Discovery 1, 15007-15018.

(2) Zhang, A., Lu, P., Dahl-Roshak, A. M., Paress, P. S., and An, Z. (2003) Efficient disruption of a polyketide synthase gene (pks1) required for melanin synthesis through Agrobacterium-mediated transformation of Glarea lozoyensis, Mol. Genet. Genomics $268,645-655$.

(3) Mattay, J., Houwaart, S., and Hüttel, W. (2018) Cryptic production of trans-3hydroxyproline in Echinocandin B biosynthesis, Appl. Environ. Microbiol. 84, e02370 